\title{
Statistical power in complex experimental designs
}

\author{
DRAKE R. BRADLEY and RONALD L. RUSSELL \\ Bates College, Lewiston, Maine \\ and \\ CHARLES P. REEVE \\ Westinghouse Savannah River Company, Aiken, South Carolina
}

\begin{abstract}
We show that power and sample size tables developed by Cohen (1988, pp. 289-354, 381-389) produce incorrect estimates for factorial designs: power is underestimated, and sample size is overestimated. The source of this bias is shrinkage in the implied value of the noncentrality parameter, $\lambda$, caused by using Cohen's adjustment to $n$ for factorial designs (pp. 365 and 396). The adjustment was intended to compensate for differences in the actual versus presumed (by the tables) error degrees of freedom; however, more accurate estimates are obtained if the tables are used without adjustment. The problems with Cohen's procedure were discovered while testing subroutines in DATASIM 1.2 for computing power and sample size in completely randomized, randomized-blocks, and split-plot factorial designs. The subroutines give the user the ability to generate power and sample size tables that are as easy to use as Cohen's, but that eliminate the conservative bias of his tables. We also implemented several improvements relative to "manual" use of Cohen's tables: (1) Since the user can control the specific values of $1-\beta, n$, and $f$ used on the rows and columns of the table, interpolation is never required; (2) exact as opposed to approximate solutions for the noncentral $F$ distribution are employed; (3) solutions for factorial designs, including those with repeated measures factors, take into account the actual error degrees of freedom for the effect being tested; and (4) provision is made for the computation of power for applications involving the doubly noncentral $F$ distribution.
\end{abstract}

Studies conducted over the last several decades show that behavioral scientists consistently neglect statistical power in the planning of research, as indicated by the low nominal power of the studies actually published (Cohen, 1962; Rossi, 1982, 1990; Sedlmeier \& Gigerenzer, 1989). Owing in large measure to the cumulative influence of Jacob Cohen's book Statistical Power Analysis for the Behavioral Sciences (Cohen, 1969, 1977, 1988), issues surrounding the determination of statistical power are finally attracting the attention of researchers. Cohen's book presents power and sample size tables for the majority of statistical tests employed by social scientists. In contrast to traditional tables, his tables tabulate power and sample size as a function of effect size. For a one-way analysis of variance with $k$ groups and $n$ observations per group, the measure of effect size is $f=\sqrt{\sum\left(\mu_{j}-\mu\right)^{2} / k \sigma_{\varepsilon}^{2}}$. Conventional power analysis, on the other hand, employs the noncentrality parameter $\lambda=N f^{2}=n k f^{2}=n \Sigma\left(\mu_{j}-\mu\right)^{2} / \sigma_{\varepsilon}^{2}$. An advantage of Cohen's approach is that $f$ (unlike $\lambda$ ) is independent of sample size $(n)$, and this simplifies both the determination of power and the organization of the

The authors thank David Howell, Richard Lehman, Glenn Meyer, Patrick Onghena, Martin Richter, and Robert Schutz for helpful comments and suggestions on an initial draft of this paper. Any errors that remain are the responsibility of the authors. Charles Reeve may be reached at 3 Spyglass Drive, Aiken, SC 29803 (e-mail: charles.reeve@) srs.gov). Reprint requests should be sent to D. R. Bradley, Department of Psychology, Bates College, Lewiston, ME 04240 (e-mail: dbradley@ abacus.bates.edu). power tables. Furthermore, the researcher can easily conceptualize $f$ in terms of a variance-explained measure, such as $\eta^{2}$ (Cohen, 1988, pp. 281-284). Since $f=\sqrt{\eta^{2} /\left(1-\eta^{2}\right)}$, power can be determined for effects corresponding to particular values of $\eta^{2}$ without a researcher's needing to know (or estimate) the values of $\mu_{j}$ and $\sigma_{\varepsilon}^{2}$. The researcher can also use Cohen's operational definitions of small $(f=.10)$, medium $(f=.25)$, and large $(f=.40)$ effects as guides for power and sample size determination (pp. 284-288). However $f$ is determined, it is a straightforward matter to estimate power or sample size for a particular application: one simply enters the appropriate table and reads the solution, interpolating where necessary.

In the present paper, we report enhancements to a statistical simulation package (DATASIM 1.2; see Bradley, 1988, 1989; Bradley \& Fleisher, 1994; Wright, 1993) that allow the researcher to produce customized power and sample size tables. It was our goal to preserve the simplicity and ease of use of Cohen's tables while at the same time making a number of improvements. First, to eliminate the need for interpolation, we give the user the option to specify the values of $n$ and $f$ (for power tables) or $1-\beta$ and $f$ (for sample size tables) to be used on the rows and columns of the table. Second, the solutions output in the tables are exact rather than approximate. This requires exact computation of the CDF of the noncentral $F$ distribution (Reeve, 1986). Third, the solutions in the tables take into account experimental design. Since Cohen's tables were developed for a one-way, completely randomized design, an adjustment is necessary $\left(n^{\prime}\right)$ for factorial de- 
signs. As we show below, this adjustment yields conservative estimates of power. With our approach, the user specifies the experimental design and the effect of interest explicitly, and exact solutions (based on the appropriate error degrees of freedom [error $d f$ ] for the effect) are displayed in the tables. Finally, we provide the capability to compute power for applications involving the doubly noncentral $F$ distribution. These topics, and demonstrations of the program output, are reviewed in the remaining sections of this paper. ${ }^{1}$

\section{DATASIM Power and Sample Size Tables}

Conventional power tables tabulate $1-\beta$ for the analysis of variance (ANOVA) as a function of the numerator $\left(v_{1}\right)$ and denominator $\left(v_{2}\right)$ degrees of freedom, the noncentrality parameter $(\lambda$ or $\phi)$, and the level of significance $(\alpha)$. The power tables developed by Cohen $(1988$, pp. 289-354) present $1-\beta$ as a function of $n, f, v_{1}$, and $\alpha$. Subtables are provided for each combination of $v_{1}$ and $\alpha$ and are organized with $n$ on rows, $f$ on columns, and $1-\beta$ in the cells. The sample size tables (pp. 381-389) follow the same format, except that $n$ appears in the cells of each table and $1-\beta$ appears on rows. Two DATASIM commands, ESP and ESN, were developed to produce power and sample size tables having the same organization as Cohen's tables. Exhibit 1 shows a power table output by ESP. The command requires the user to specify the distribution $(F)$, number of conditions $(k=4)$, design ( $S / T=$ independent groups), and $\alpha$ level $(\alpha=.05)$. The user may also specify iteration sequences ${ }^{2}$ to control the values of $n$ and $f$ employed in the rows and columns of the table. If none are supplied, DATASIM uses $10(10) 100$ for $n$ and .1(.1). 8 for $f$. The iteration sequences in Exhibit 1 produce a power table identical to Cohen's Table 8.3.14 (p. 315). To conserve space, only the first and last 3 rows (out of 75) are shown. The corresponding entries in the two tables are in close agreement. The differences that exist are due to the fact that Cohen's table displays approximate power to two decimal places, whereas the ESP table displays exact power to four decimal places.

Exhibit 2 illustrates a sample size table generated by the ESN command for the same example. The syntax of the command follows the ESP command, except that the first iteration sequence controls the values of $1-\beta$ (rather than $n$ ) assigned to the rows of the table (the default sequence is .1(.1).9/.99). The table in Exhibit 2 is identical to Cohen's Table 8.4.4 ( $u=3$, p. 384). There is again very close agreement between the values in the two tables. Different conventions account for some minor discrepancies: Cohen rounds up the fractional values of $n$ obtained in his solutions, whereas DATASIM rounds to the nearest integer.

The information in power and sample size tables is the same: the only difference is the parameter $(1-\beta$ or $n)$ assigned to the cell as opposed to the row of the table. The two tables should therefore give identical results for a given combination of $f, v_{1}$, and $\alpha$. Since Cohen's power tables are based on an approximation to the noncentral $F$ distribution (p. 550), whereas his sample size tables are based on exact tables of power points obtained from NBS (p. 551), small inconsistencies sometimes arise. The ESP and ESN commands employ the same (exact) algorithm for computing the CDF of the noncentral $F$ distribution. Consequently, the only discrepancies that arise are those attributable to the fact that ESN displays sample sizes rounded to the nearest integer value. Once such discrepancy occurs at $\{n=3, f=.30\}$, for which Exhibits 1 and 2 indicate power of .0942 and .10 , respectively. The NSAMP command may be used to display the exact value of $n$ that was rounded to $n=3$ in Exhibit 2. The format for NSAMP is the same as that for ESN, except that the user must additionally specify the power $(1-\beta)$ to be used in calculating the sample size. Exhibit 3 illustrates NSAMP for the case under consideration: $k=4, S / T, \alpha=.05,1-\beta=.10$, and $f=.30$. After selecting a starting value of $n=2$, NSAMP successively doubles the $n$ until two values are found which have power values bracketing $1-\beta=.10$. Only one doubling is required in this case. Next, a binary search is conducted within this interval to find the $n$ that gives $1-\beta=.10$ exactly. This turns out to be $n=3.2277$. This exact $n$ was rounded to 3 by the ESN command in Exhibit 2; hence the apparent discrepancy with Exhibit $1 .{ }^{3}$

Execution of the ESP and ESN commands requires a fair amount of time. ${ }^{4}$ This is particularly true of ESN, which executes the binary search procedure employed by

- ESP F S S/T.05 2(1)40(2)60(4) $100(20) 200(50) 500(100) 1000.05(.05) .41 .1) .8$, BEEP

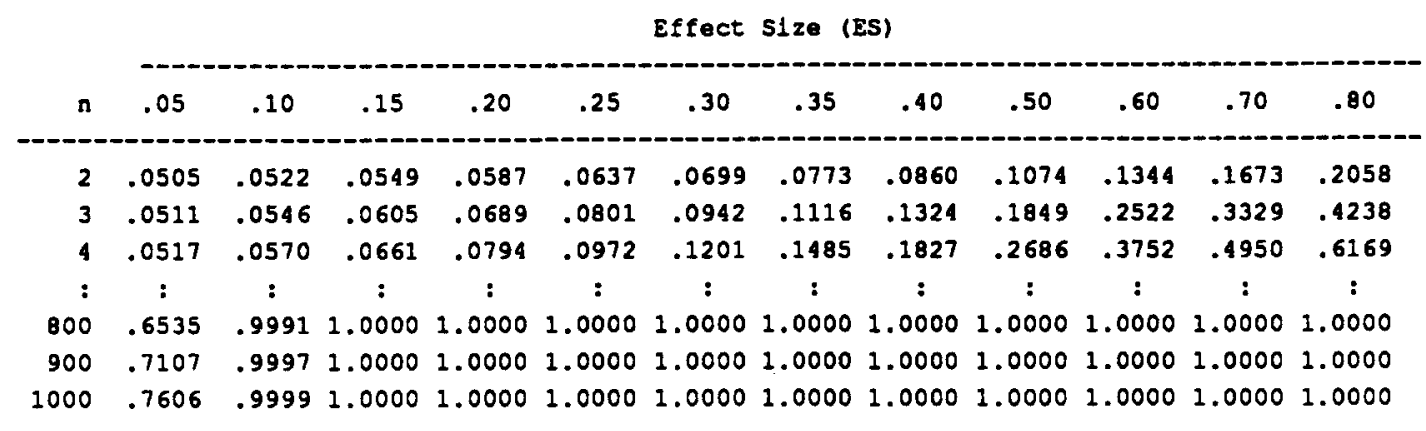

Note: tabled entrles are power $(1-B)$.

Exhibit 1. Illustration of the ESP command for a one-way completely randomized design. 
ESN F \& S/T .05 $.1 / .5 / .7(.1) .9 / .95 / .99 \quad .05(.05) .4(.1) .8$, BEEP

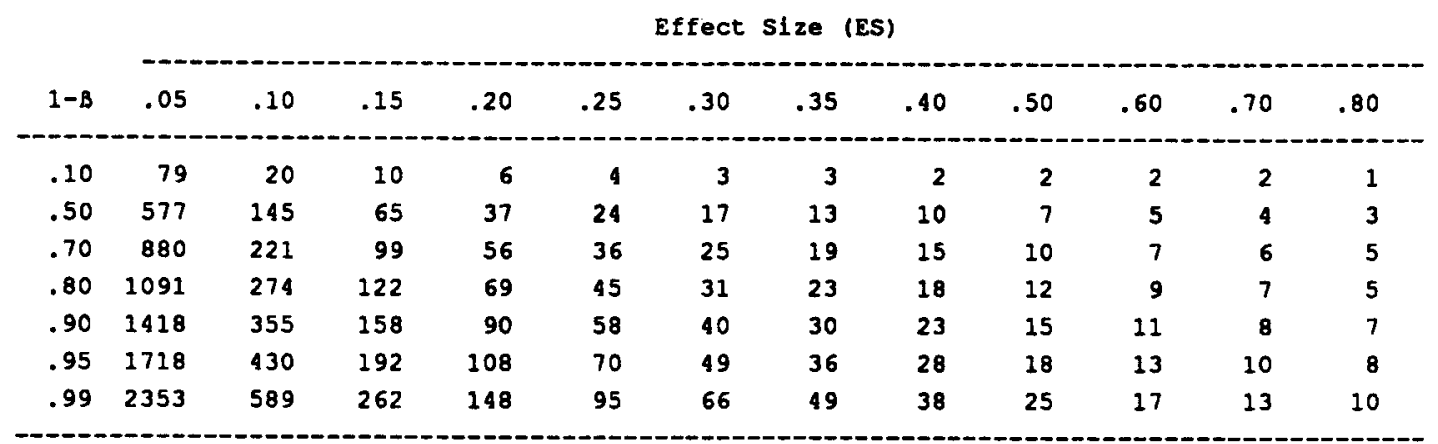

Note: tabled entries are sample size $(n)$.

Exhibit 2. Illustration of the ESN command for a one-way completely randomized design.

NSAMP for each and every cell of the table. Since the information contained in power and sample size tables is the same, and iteration sequences allow as fine a "grain" on rows as desired, the ESP command is recommended over the ESN command whenever execution time is at issue. In either case, the BEEP command can be appended as shown in Exhibits 1 and 2, and when all of the entries of the table have been computed, DATASIM will alert the user by sounding the system "beep" repeatedly. Pressing any key will terminate the beep and display the table.

DATASIM can also compute power using the "conventional" approach. This is accomplished by the POWER command. For applications involving ANOVA, the format is: POWER F $\left\langle v_{1}\right\rangle\left\langle v_{2}\right\rangle\langle\alpha\rangle\langle\lambda\rangle$. Since $v_{1}$ and $v_{2}$ are specified explicitly, there is no need to indicate the dimensions $(n, k)$ and type $(S / T)$ of design. To illustrate, Exhibit 1 shows that the power for $n=4$ and $f=.80$ (where $k=4$ and $\alpha=.05$ ) is .6169 . To compute power using the conventional approach, we find $v_{1}=k-1=3, v_{2}=$ $k(n-1)=4(3)=12$, and $\lambda=N f^{2}=n k f^{2}=4(4) .8^{2}=$ 10.24, and entering POWER F 312.0510 .24 gives $1-\beta=.6169$. By specifying iteration sequences for $v_{1}$, $v_{2}, \alpha$, and/or $\lambda$, the user can have DATASIM generate tables that display power as a function of these parameters.

\section{Exact Versus Approximate Solutions}

In developing the computational algorithms for ESP, ESN, NSAMP, and POWER, we reviewed several approximations to the CDF of the noncentral $F$ distribution. Laubscher's $(1960$, p. 1111) square root and cube root normal approximations, used by Cohen (1988, p. 550) in the construction of his power tables, were briefly considered. Except for very small $n$ and $v_{2}$, these approximations are generally accurate to \pm .02 of the true value. We also considered the two-moment (Patnaik, 1949, p. 221) and three-moment (Tiku, 1965, p. 425) approximations. Extensive testing using

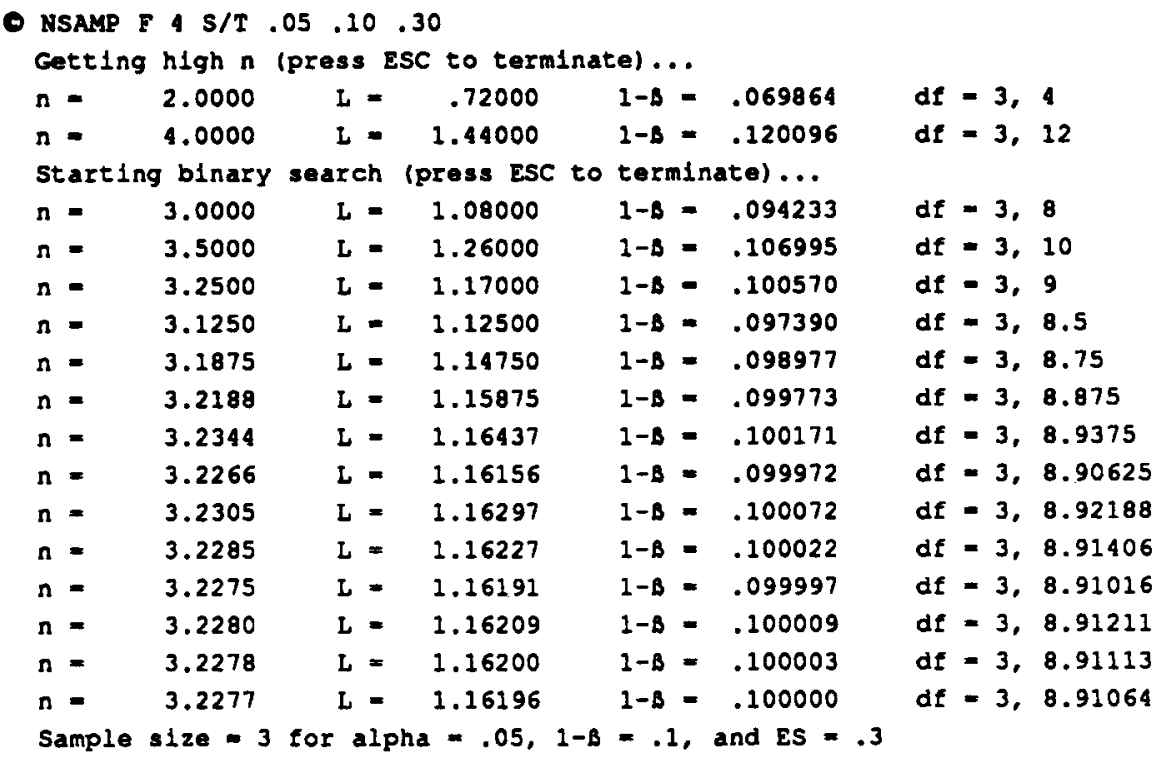

Exhibit 3. Illustration of the NSAMP command for a one-way completely randomized design. $L$ is the noncentrality parameter, $\lambda$. 
the 7,920 entries in Tiku's power tables as benchmarks (Tiku, 1967, pp. 527- 537) revealed that the three-moment approximation was the most accurate of the four approximations, remaining within \pm .008 of the true value (Bradley, Russell, \& Reeve, 1996). However, the three-moment approximation was not noticeably faster or more efficient than algorithms developed by one of the authors for computing the CDF of singly or doubly noncentral $F$ to a specified precision (Reeve, 1986). Consequently, we decided to employ exact rather than approximate methods for computing power and sample size.

Researchers in the behavioral sciences are familiar with the singly noncentral $F$ distribution, denoted $F^{\prime}$. This is the distribution of the ratio of a noncentral $\chi^{2}$ divided by its degrees of freedom to a central $\chi^{2}$ divided by its degrees of freedom. Tables of noncentral $F$ for $\left\{v_{1}, v_{2}, \lambda\right\}$ are provided in many experimental design texts. In contrast, the doubly noncentral $F$, denoted $F^{\prime \prime}$, is less commonly encountered. This is the distribution of the ratio of one noncentral $\chi^{2}$ divided by its degrees of freedom to a second noncentral $\chi^{2}$ divided by its degrees of freedom:

$$
Y=\frac{\chi_{1}^{2} / v_{1}}{\chi_{2}^{2} / v_{2}}
$$

The random variable $Y$ exhibits a doubly noncentral $F$ distribution with parameters $\left\{v_{1}, v_{2}, \lambda_{1}, \lambda_{2}\right\}$. The distribution reduces to singly noncentral $F$ for $\lambda_{2}=0$, and to central $F$ for $\lambda_{1}=\lambda_{2}=0$. Consequently, algorithms for computing the CDF of $F^{\prime \prime}$ can supply solutions for $F^{\prime}$ and $F$ as well. Exhibit 4 shows a series representation of the cumulative distribution function of $F^{\prime \prime}$ (Reeve, 1986). $A_{i}$ and $B_{j}$ are Poisson probabilities, and $I(u, a, b)$ is the CDF of the beta distribution (also called the incomplete beta ratio). Evaluation of $F^{\prime \prime}$ requires subroutines to calculate the gamma function and the beta CDF. Indices for summing the Poisson probabilities also have to be determined, since, for computational purposes, the infinite series must be truncated. FORTRAN subroutines written by Reeve (1986) while at the National Bureau of Standards (now the National Institute of Science and Technology) were opti- mized to perform these tasks. With respect to the indices of summation, Reeve (1986) notes that by selecting values for $I^{\prime}, I^{\prime \prime}, J^{\prime}$, and $J^{\prime \prime}$ which result in

$$
\sum_{i=I^{\prime}}^{I^{\prime \prime}} A_{i} \geq 1-\frac{\varepsilon}{2} \text { and } \sum_{i=J^{\prime}}^{J^{\prime \prime}} B_{j} \geq 1-\frac{\varepsilon}{2},
$$

$\varepsilon$ serves as an absolute error bound on $F_{Y}(x)$, with $\varepsilon$ being specified by the researcher as the maximum tolerable error in the solution. The CDF of $F^{\prime \prime}$ can therefore be computed with an accuracy that is limited only by the available machine precision. To reduce computation time, recurrence relations (Abramowitz \& Stegun, 1970, p. 944) are used to limit the number of direct evaluations of the beta CDF. Reeve's algorithms are employed by the ESP, ESN, NSAMP, and POWER commands. The reader is referred to Reeve (1986) for additional details.

\section{Repeated Measures and Factorial Designs}

The power and sample size tables in Cohen (1988) assume a one-way independent groups design. If the researcher anticipates using a repeated measures or matchedgroups design, the tables will overestimate power somewhat because the error $d f$ is assumed to be $v_{2}=k(n-1)$ rather than $v_{2}=(n-1)(k-1)$. Cohen points out that the difference in the solutions obtained will be "trivial for all but small samples" (p. 49). Our own checks confirm this observation. For the specific example cited by Cohen (p. 50), where $k=2, n=40$, and $f=.395(d=.79)$, we used the following variants of the ESP command to generate single-entry power tables:

ESP F 2 S/T $.05 / 40 / .395$, ESP F 2 TxS $.05 / 40 / .395$.

The first variant $(S / T)$ computes exact power for $v_{2}=$ $2(40-1)=78$, and the second $(T \times S)$ does the same for $v_{2}=(40-1)=39$. The resulting power values are .9369 and .9312. As Cohen claims, the difference is indeed trivial. The difference is a bit more substantial for $n=10$ : .3874 and .3524 , respectively. To assure exact solutions, the ESP, ESN, and NSAMP commands require the user to specify the design as $S / T$ (independent groups) or

$$
\begin{aligned}
& F_{Y}(x)=\sum_{i=0}^{\infty} \sum_{j=0}^{\infty} A_{i} B_{j} I\left(u, \frac{v_{1}}{2}+i, \frac{v_{2}}{2}+j\right), \quad \text { where: } \\
& A_{i}=\frac{\left(\frac{\lambda_{1}}{2}\right)^{i} e^{-\lambda_{1} / 2}}{\Gamma(i+1)}, \quad B_{j}=\frac{\left(\frac{\lambda_{2}}{2}\right)^{j} e^{-\lambda_{z} / 2}}{\Gamma(j+1)}, \quad u=\frac{v_{1} x}{\left(v_{1} x+v_{2}\right)}, \\
& I(u, a, b)=\int_{0}^{\frac{t^{a-1}(1-t)^{b-1}}{B(a, b)} d t, \quad B(a, b)=\int_{0}^{t} t^{a-1}(1-t)^{b-1} d t}
\end{aligned}
$$


$T \times S$ (correlated groups). This allows DATASIM to employ the proper $d f$ in computing the solution.

A similar issue arises for factorial designs. Following Cohen (1988, pp. 367-368), consider a $3 \times 4(p \times q)$ independent-groups design with $n=6$ per cell $(N=72$ overall). The error $d f$ for the two-way ANOVA is $v_{2}=$ $N-p q=60$. To compute power for the main effect of $A$ using Cohen's tables, we proceed as if factor $A$ were being investigated in a one-way design with $n_{A}=n q$ observations per group. ${ }^{5}$ In doing this, however, the implicit error $d f$ for factor $A$ is $v_{2}=N-p=69$. The increase in $v_{2}$ occurs because the tables do not take into account the reduction in the error $d f$ that results from estimating the effects of $B(3 d f)$ and the $A B$ interaction (6 df) in the two-way ANOVA. The same problem arises for factor $B$, which has an implicit error $d f$ of $v_{2}=N-q=68$. Solutions based on these "inflated" error $d f$ s will therefore overestimate true power (and underestimate required sample size). To prevent this, Cohen (p. 365) recommends adjusting the $n$ used to enter his power tables as follows:

$$
n^{\prime}=\frac{v_{2}}{v_{1}+1}+1, \quad n_{A}^{\prime}=\frac{v_{2}}{p}+1, \quad n_{B}^{\prime}=\frac{v_{2}}{q}+1 .
$$

The first equation is for the general case, and the second two are for factors $A$ and $B$ in a two-way design. The adjusted $n$ s for the present example are $n_{A}^{\prime}=60 / 3+1=$ 21 and $n_{B}^{\prime}=60 / 4+1=16$. For comparison, the unadjusted values are $n_{A}=n q=24$ and $n_{B}=n p=18$. The error $d f$ implicitly associated with the adjusted $n \mathrm{~s}$ is $p\left(n_{A}^{\prime}-1\right)=3(21-1)=60$ and $q\left(n_{B}^{\prime}-1\right)=4(16-1)=60$. This shows that the adjustment does indeed correct for the inflated $d f$ which would otherwise arise from using Cohen's tables with $n_{A}$ or $n_{B}$.

Using adjusted $n$ s of $n_{A}^{\prime}=21$ and $n_{B}^{\prime}=16$, and assuming respective effect sizes of $f_{A}=.25$ and $f_{B}=.40$, Tables 8.3.13 and 8.3.14 in Cohen (1988) show the power to be .40 and .75 , respectively. To check these values against those obtained by a conventional analysis using the POWER command, we need to compute the noncentrality parameters $\lambda_{A}$ and $\lambda_{B}$ :

$$
\begin{aligned}
& \lambda_{A}=N f_{A}^{2}=\frac{n p q \sum\left(\mu_{i}-\mu\right)^{2}}{p \sigma_{\varepsilon}^{2}}=\frac{n q \sum\left(\mu_{i}-\mu\right)^{2}}{\sigma_{\varepsilon}^{2}}, \\
& \lambda_{B}=N f_{B}^{2}=\frac{n p q \sum\left(\mu_{j}-\mu\right)^{2}}{q \sigma_{\varepsilon}^{2}}=\frac{n p \sum\left(\mu_{j}-\mu\right)^{2}}{\sigma_{\varepsilon}^{2}} .
\end{aligned}
$$

Note that for the two-way design under consideration, $\lambda_{A}=N f_{A}^{2}=p n_{A} f_{A}^{2}$ and $\lambda_{B}=N f_{B}^{2}=q n_{B} f_{B}^{2}$. Now, we can use either the adjusted $\left(n_{A}^{\prime}, n_{B}^{\prime}\right)$ or unadjusted $\left(n_{A}, n_{B}\right)$ $n \mathrm{~s}$ to compute the noncentrality parameters, and doing it both ways gives

$$
\begin{aligned}
\lambda_{A}^{\prime} & =p n_{A}^{\prime} f_{A}^{2}=3(21)(.25)^{2} & \lambda_{B}^{\prime} & =q n_{B}^{\prime} f_{B}^{2}=4(16)(.40)^{2} \\
& =3.9375 & & =10.24 \\
\lambda_{A} & =p n_{A} f_{A}^{2}=3(24)(.25)^{2} & \lambda_{B} & =q n_{B} f_{B}^{2}=4(18)(.40)^{2} \\
& =4.50 & & =11.52
\end{aligned}
$$

For the adjusted $\lambda \mathrm{s}$, POWER $\mathrm{F} 260.053 .9375$ and POWER F 360.0510 .24 compute the power of $A$ and $B$ as .3920 and .7417 , respectively. These values agree with those obtained above (.40 and .75) using Cohen's tables. For the unadjusted $\lambda \mathrm{s}$, however, POWER F 260.054 .50 and POWER F 360.0511 .52 yield values of .4409 and .7955 , respectively. Now, since the latter values were obtained using the correct error $d f\left(v_{2}=60\right)$, the standard formula for the noncentrality parameter $\left(\lambda=N f^{2}\right)$, and an exact algorithm for computing power (Reeve, 1986), they are in fact the true power for the tests on factors $A$ and $B$. We conducted a DATASIM simulation to confirm this. The simulation generated 10,000 datasets for a $3 \times 4$ factorial design with $n=6$ observations per cell and effect sizes of $f_{A}=.25$ and $f_{B}=.40$ (see the Appendix). The proportion of $F \mathrm{~s}$ with $p \leq .05$ was .4414 for factor $A$ and .7930 for factor $B$. These proportions do not differ significantly from the (unadjusted) DATASIM power values of .4409 and $.7955: z=.10, p=.92$, and $z=$ $-.62, p=.56$. In contrast, they both differ significantly from the power values of .40 and .75 obtained using adjusted $n$ s and Cohen's tables: $z=8.45, p<.0001$, and $z=$ $9.93, p<.0001$.

The purpose of Cohen's adjustment to $n$ is to offset the inflation in the error $d f$ which occurs when using his tables for higher order designs. While the adjustment does produce nominally correct error $d f$ for the tests, it has the side effect of reducing the implied $N$ for the overall analysis, and this reduces the value of $\lambda$. The result is a conservative bias in the $n$-adjusted power estimates for factorial designs. It is worth noting that a similar issue was present above, when we discussed the fact that Cohen's tables do not explicitly accommodate the reduced $d f$ for the error terms employed by repeated measures designs. As Cohen rightly points out, the bias produced by this is trivial (except for small samples), and he therefore makes no attempt to "adjust" for it. As it happens, the bias which arises when one uses Cohen's tables for factorial designs (without adjusting for inflated $d f$ ) is also trivial. We can show this by entering Table 8.3.13 with $n_{A}=24$ rather than $n_{A}^{\prime}=21$, and Table 8.3.14 with $n_{B}=18$ rather than $n_{B}^{\prime}=16$. The resulting power is .45 for factor $A$ and .80 for factor $B$. These values only slightly overestimate the true power of .4409 and .7955 .

The discrepancies between $n$-adjusted and true power should increase as the number of factors and the number of levels per factor increases. Cohen's Table 8.3.34 (p. 375) presents $n$-adjusted power for the main and interaction effects in a $3 \times 4 \times 5$ design with $n=3$ observations per cell, $f=\{.10, .25, .40\}$, and $\alpha=\{.01, .05, .10\}$. Table 1 reproduces Cohen's results for $\alpha=.05$, along with the exact solutions output by DATASIM. As expected, the $n$-adjusted values consistently underestimate actual power. For $f=.25$ the $n$-adjusted power for factor $A$ is .70 , whereas the actual power is .8516 . The underestimation is due to the "shrinkage" in the overall sample size, $N^{\prime}=$ $p n_{A}^{\prime}=(3) 41=123$, relative to the actual value of $N=$ $p n_{A}=(3) 60=180$. This produces a corresponding dif- 
ference in the noncentrality parameters, $\lambda_{A}^{\prime}=N^{\prime} f^{2}=$ $123(.25)^{2}=7.6875$ and $\lambda_{A}=N f^{2}=180(.25)^{2}=11.25$. Now, for $\left\{v_{1}=2, v_{2}=120, \lambda_{A}^{\prime}=7.6875\right\}$, the DATASIM POWER command indicates that $1-\beta=.6875$, which is close to the $n$-adjusted value of . 70 obtained by Cohen. ${ }^{6}$ This shows that the power values in Cohen's Table 8.3.34 are indeed based on shrunken $\lambda$ s. Conversely, if we enter Cohen's Table 8.3.13 with $f=.25$ and the unadjusted $n$ for factor $A$-that is, with $n_{A}=n q r=60$ rather than $n_{A}^{\prime}=41$-we find that factor $A$ has power of .86 . This "unadjusted" estimate only slightly overestimates the true power of .8516 in Table 1. These examples argue against making Cohen's adjustment to $n$ when one uses his power and sample size tables for factorial designs.

One of the readers of an initial draft of this paper, Patrick Onghena, pointed out that the conservative bias associated with Cohen's $n$-adjustment procedure was first noted in a footnote to a paper by Koele (1982, note 1). In an unpublished technical report, Koele and Hoogstraten (1980) analyze the problem in much greater detail and show (p. 9) that Cohen's tables can be used for factorial designs with $n^{\prime}$, provided that one also adjusts $f$ as follows:

$$
f^{\prime}=f \sqrt{\frac{N}{v_{1}+v_{2}+1}}=f \sqrt{\frac{N}{N^{\prime}}} .
$$

Koele and Hoogstraten's adjustment to $f$ thus compensates for the shrinkage in $\lambda$ caused by the reduction in the overall sample size, $N^{\prime}$ versus $N$, which results from using $n^{\prime}$ rather than $n$ in the tables. The value of $f^{\prime}$ is adjusted upward in proportion to the difference between $N^{\prime}$ and $N$, and this offsets the conservative bias that would otherwise arise with Cohen's tables. Onghena (1995, personal communication) also alerted us to the paper by Erdfelder, Faul, and Buchner (1996) which addresses the $n$-adjustment problem and Koele's initial work on this issue. Like the present authors, Erdfelder et al. apparently uncovered the errors in Cohen's approach while testing algorithms for computing power and sample size in their program GPOWER. It is of some interest that the $n$-adjustment problem, noted in passing by Koele in a footnote to his 1982 paper, has remained largely unknown to researchers and investigators, and apparently to Cohen himself, since the error is still present in the 1988 edition of his book. This is surprising, because the magnitude of the bias can be quite large. For the example noted above, the $n$-adjusted versus actual power for $F_{A}$ in a $3 \times 4 \times 5$ design with $n=3$ and $f_{A}=.25$ was .70 versus .8516 . If we work the problem backwards, and ask the true sample size required to achieve a power of $1-\beta=.70$, we find that $n=2.1458$ is needed, not $n=3$ as implied by Cohen's tables. The difference in the overall sample sizes is $N \approx 129$ versus $N=180$. Cohen's solution would therefore cause the researcher to use a sample size that is $40 \%$ larger than necessary to achieve power of $1-\beta=.70$.

The preceding discussion has reviewed some of the complications that arise when one attempts to make one set of power and sample size tables work for a large variety of experimental designs. This is the reason the DATASIM ESP, ESN, and NSAMP commands require the user to specify the experimental design and the effect $(A, B$, $A B)$ explicitly. Doing this permits DATASIM to compute the proper error $d f$ for the effect, and to calculate exact (not approximate) power. To illustrate, suppose we wish to display a power table for the $A B$ interaction in a $2 \times$ $3 \times 2$ factorial design with repeated measures on $B$. This is done by entering $\mathrm{ESP} F 2 \times 3 \times 2 \mathrm{BxS} / \mathrm{AC} \mathrm{AB} .05$. The design token "BxS/AC" signifies that $A$ and $C$ are betweengroups factors and $B$ is a within-groups factor. The token "AB" tells DATASIM to compute power for the $A B$ interaction effect. At present, DATASIM accommodates designs having any mixture of up to three independentand/or correlated-groups factors.

\section{The Doubly Noncentral $F$ Distribution}

We noted above that the algorithms developed by Reeve (1986) compute power for the doubly noncentral $F$ distribution. Scheffé (1959, pp. 134-136) gives an example of an application in which this distribution arises. Suppose that a researcher intends to employ a $2 \times 3$ factorial design with $n=1$ observations per cell. In this case, tests on the main effects of $A$ and $B$ employ $M S_{A B}$ as the error term, because $M S_{W C E L L}$ cannot be computed. The researcher hopes that $A B$ interaction variance is either absent or minimal; otherwise tests of the main effects will suffer a loss in power. (There is, of course, the additional complication that interpretation of the $A$ and $B$ main effects is qualified by the presence of an $A B$ interaction in the data.) Nevertheless, in computing power the researcher should consider the possibility that nonzero interaction variance $\left(\lambda_{A B}>0\right)$

Table 1

A Comparison of $\boldsymbol{n}$-Adjusted and Exact Power for the Main and Interaction Effects in a $3 \times 4 \times 5$ Factorial Design With $n=3$ Observations per Cell $(\alpha=.05)$

\begin{tabular}{|c|c|c|c|c|c|c|c|c|}
\hline \multirow[b]{2}{*}{ Effect } & \multirow[b]{2}{*}{$v_{1}$} & \multirow[b]{2}{*}{$n^{\prime}$} & \multicolumn{3}{|c|}{$n$-Adjusted $1-\beta$} & \multicolumn{3}{|c|}{ Exact $1-\beta$} \\
\hline & & & $f=.10$ & $f=.25$ & $f=.40$ & $f=.10$ & $f=.25$ & $f=.40$ \\
\hline A & 2 & 41 & .15 & .70 & .98 & .2023 & .8516 & .9986 \\
\hline B & 3 & 31 & .13 & .63 & .97 & .1718 & .7995 & .9970 \\
\hline C & 4 & 25 & .12 & .58 & .96 & .1529 & .7541 & .9947 \\
\hline $\mathrm{AB}$ & 6 & 18.1 & .10 & .51 & .93 & .1301 & .6781 & .9882 \\
\hline $\mathrm{AC}$ & 8 & 14.3 & .09 & .46 & .91 & .1165 & .6164 & .9792 \\
\hline $\mathrm{BC}$ & 12 & 10.2 & .08 & .39 & .86 & .1007 & .5217 & .9550 \\
\hline $\mathrm{ABC}$ & 24 & 5.8 & .08 & .29 & .74 & .0811 & .3585 & .8527 \\
\hline
\end{tabular}


will cause $F_{A}$ and $F_{B}$ to follow the doubly noncentral $F$ distributions $F\left(v_{A}, v_{A B}, \lambda_{A}, \lambda_{A B}\right)$ and $F\left(v_{B}, v_{A B}, \lambda_{B}, \lambda_{A B}\right)$, respectively. Given the relation $\lambda=N f^{2}$, it is a straightforward matter to compute different values of $\lambda$ for different assumed effect sizes. In the absence of other information, Cohen's conventions for small, medium, and large effects can be employed for $f_{A}, f_{B}$, and $f_{A B}$, and a matrix of possible values of $\lambda_{A}, \lambda_{B}$, and $\lambda_{A B}$ determined. The POWER command may then be used to compute the power of $F_{A}$ and $F_{B}$, but with one modification: a second $\lambda$ parameter must be supplied. The format is POWER F $\left\langle v_{1}\right\rangle\left\langle v_{2}\right\rangle$ $\langle\alpha\rangle\left\langle\lambda_{1}\right\rangle\left\langle\lambda_{2}\right\rangle$ for the general case, and for tests on factors $A$ and $B$ in designs with $n=1$ per cell:

$$
\begin{aligned}
& \text { POWER F } \left.\left\langle v_{A}\right\rangle\left\langle v_{A B}\right\rangle\langle\alpha\rangle\left\langle\lambda_{A}\right\rangle<\lambda_{A B}\right\rangle, \\
& \text { POWER F } \left.<v_{B}\right\rangle\left\langle v_{A B}\right\rangle\langle\alpha\rangle\left\langle\lambda_{B}\right\rangle\left\langle\lambda_{A B}\right\rangle .
\end{aligned}
$$

The researcher can thereby assess the power of $F_{A}$ and $F_{B}$ in the presence of varying degrees of $A B$ interaction variance.

\section{Summary}

This paper has reviewed a number of issues with respect to statistical power in complex experimental designs. Two recently developed DATASIM commands, ESP and ESN, preserve the simplicity and ease of use of Cohen's (1988) power and sample size tables, and obviate certain problems that arise when those tables are used for repeated measures or factorial designs. Furthermore, the availability of efficient algorithms for computing exact power (Reeve, 1986) makes it unnecessary to rely on approximations. Exact algorithms are supplemented in DATASIM by routines which compute the proper error $d f$ for the effects of interest in completely randomized, randomized-blocks, and split-plot factorial designs having up to three factors. The resulting power values are accurate to at least seven or eight digits and are limited only by the available machine precision.

\section{REFERENCES}

Abramowitz, M., \& Stegun, I. A. (EDS.) (1970). Handbook of mathematical functions (NBS Special Publication No. 55). Washington, DC: National Bureau of Standards.

BradLEy, D. R. (1988). DATASIM. Lewiston, ME: Desktop Press.

BRADley, D. R. (1989). Computer simulation with DATASIM. Behavior Research Methods, Instruments, \& Computers, 21, 99-112.

BRADLEY, D. R., \& FLEISHER, C. L. (1994). Generating multivariate data from nonnormal distributions: Mihal and Barrett revisited. Behavior Research Methods, Instruments, \& Computers, 26, 156-166.

Bradley, D. R., Russell, R. L., \& Reeve, C. P. (1996). The accuracy of four approximations to noncentral $F$. Manuscript submitted for publication.

CoHEN, J. (1962). The statistical power of abnormal-social psychological research: A review. Journal of Abnormal \& Social Psychology, 65, 145-153.

COHEN, J. (1969). Statistical power analysis for the behavioral sciences. New York: Academic Press.

COHEN, J. (1977). Statistical power analysis for the behavioral sciences (rev. ed.). New York: Academic Press.

COHEN, J. (1988). Statistical power analysis for the behavioral sciences (2nd ed.). Hillsdale, NJ: Erlbaum.

ERDFELDER, E., FAUL, F., \& BUCHNER, A. (1996). GPOWER: A general power analysis program. Behavior Research Methods, Instruments, \&
Computers, 28, 1-11.

KoElE, P. (1982). Calculating power in the analysis of variance. Psychological Bulletin, 92, 513-516.

Koele, P., \& Hoogstraten, J. (1980). Power and sample size calculations in the analysis of variance (Révész Berichten No. 12). Amsterdam: Psychologisch Laboratorium, Universiteit van Amsterdam.

LAUBSCHER, N. F. (1960). Normalizing the noncentral $t$ and $F$ distributions. Annals of Mathematical Statistics, 31, 1105-1112.

Patnaik, P. B. (1949). The non-central $\chi^{2}$ and $F$ distributions and their applications. Biometrics, 36, 202-232.

REEVE, C. P. (1986). An algorithm for computing the doubly noncentral $F$ C.D.F. to a specified accuracy (Statistical Engineering Division Note 86-4). Gaithersburg, MD: National Bureau of Standards.

Rossi, J. S. (1982). Meta-analysis, power analysis and artifactual controversy: The case of spontaneous recovery of verbal associations. Paper presented at the meeting of the Eastern Psychological Association, Baltimore.

Rossi, J. S. (1990). Statistical power of psychological research: What have we gained in 20 years? Journal of Consulting \& Clinical Psychology, 58, 646-656.

SCHEFFÉ, H. (1959). Analysis of variance. New York: Wiley.

Sedlmeier, P., \& Gigerenzer, G. (1989). Do studies of statistical power have an effect on the power of studies? Psychological Bulletin, $105,309-316$.

Tiku, M. L. (1965). Laguerre series forms of non-central $\chi^{2}$ and $F$ distributions. Biometrika, 52, 415-427.

Tiku, M. L. (1967). Tables of the power of the $F$-test. Journal of the American Statistical Association, 62, 525-539.

Wright, J. (1993). Datasim 1.0. Psychology Software News, 4(1), 15-16.

\section{NOTES}

1. Although the present paper focuses specifically on the $F$ distribution, DATASIM can also generate power and sample size tables for $z, t$, $r, \chi^{2}$, and $R^{2}$.

2. To illustrate, consider the iteration sequence 1.0(.2)2.2(.4)3.0. This is a "compressed" representation of 1.0, 1.2, 1.4, 1.6, 1.8, 2.0, 2.2, 2.6, and 3.0. The notation $R 1(R 2) R 3$ denotes a series of values that starts with $R 1$, ends with $R 3$, and increments by $R 2$. Values not conforming to the iteration pattern are separated by commas throughout the sequence: for example, $2,3,5(5) 20(10) 40(20) 100,150,200(100) 1000$. In DATASIM, commas serve as command delimiters, so commas in the iteration sequence are replaced by slashes $(/)$.

3. Although fractional $n \mathrm{~s}$ are meaningless from a practical standpoint, in that the researcher must run subjects in increments of 1 , there is nothing in the mathematics of power and sample size determination that limits solutions to integer values of $n$. In the interest of preserving exact solutions, the NSAMP command therefore computes and displays the sample size and error $d f$ as fractional values (Exhibit 3 ).

4. The tables in Exhibits 1 and 2 required 89 and $168 \mathrm{sec}$, respectively, to compute and display on a Macintosh Centris 650 (68040 with FPU). A total of $75 \times 12=900$ power values were computed for Exhibit 1 , and $7 \times 12=84$ sample sizes were computed for Exhibit 2 . Hence, computation of each power value requires about $89 / 900=.10 \mathrm{sec}$, and computation of each sample size requires $168 / 84=2.00 \mathrm{sec}$. The additional time needed for sample size computations $(2.00 / .10=20: 1)$ is due to the fact that the search required to home in on the exact value of $n$ takes about 20 iterations. In Exhibit 3, for example, two iterations were needed to bracket the desired $n$, and 14 additional iterations were required to find the exact value of $n$. The search procedure currently employed is not the most efficient: in future releases we hope to speed up sample size computations by a factor of $2: 1$ or more. (One possibility is to use the secant method to find $n$.)

5. In our notation for a $p x q$ factorial, $n$ is the sample size per cell, $n_{A}$ is the number of observations in each row of factor $A, n_{B}$ is the number of observations in each column of factor $B$, and $N$ is the total number of observations. Note that $n_{A}=n q, n_{B}=n p$, and $N=n p q=p n_{A}=q n_{B}$.

6 . The reason for the difference is that Cohen uses the square root approximation, which gives a value of 6956 to four places. The value of .6875 output by the POWER command is exact. 
APPENDIX

The following commands were used to initialize and run the DATASIM simulation:

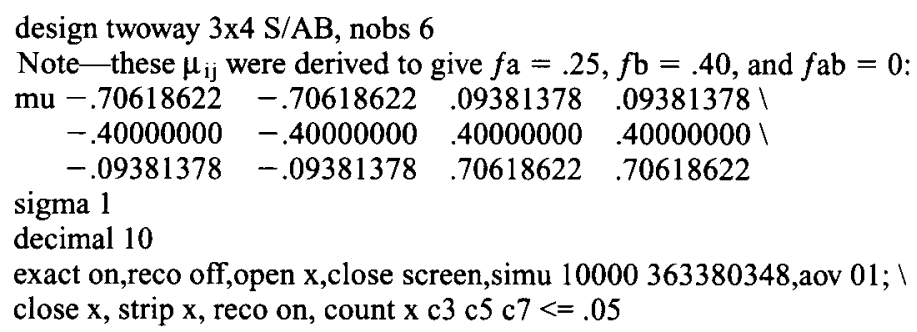

